\title{
Therapeutic efficacy of Thornton adjustable positioner in the management of patients with severe Obstructive sleep apnea : A Pilot study
}

\author{
B Jayan, BNBM Prasad, UR Kamat, OP Kharbanda, D Bhattacharrya
} Army Dental Centre (R\&R), Army Hospital (R\&R), Delhi Cantt, and AllMS, New Delhi

Indian J Sleep Med 2008; 3.3, 97-101

\begin{abstract}
Mandibular advancement appliances (MAD) have long been used to treat OSA. Most of the international sleep medicine societies recommend MAD in mild and moderate OSA. Most studies with MAD exclude patients with severe OSA and include patients who failed other treatment modalities with significant source of bias. Cephalometric and BMI factors are often not factored while prescribing MAD. So we undertook a pilot study to evaluate the therapeutic efficacy of Thornton adjustable positioner (TAP), a titratable MAD in severe adult OSA cases. We have observed significant $(p<.0001)$ improvement in Apnea-Hypopnea index and Epworth sleepiness scale. We concluded that factoring predictable cephalometric measurements, BMld $\leq 30 \mathrm{Kg} / \mathrm{m} 2$, and mandibular protrusion of $70 \%$; severe OSA can be effectively managed with TAP.
\end{abstract}

Keywords: Thornton adjustable positioner, severe Obstructive sleep apnea

\section{Introduction}

$\mathrm{O}$ bstructive sleep apnea(OSA) results in any condition or disease that causes partial or complete obstruction of airway when the patient assumes a supine position and goes to sleep. Mandibular advancement appliances have long been used to treat OSA. Their efficacy regarding respiration during sleep varies greatly and remains difficult to predict.Practice parameters for treatment of OSA with oral appliances by American sleep disorders association and German society of sleep medicine has recommended Oral appliances in mild to moderate OSA patients and in severe cases not amenable to CPAP therapy or surgery $[1,2,3]$.The effectiveness of mandibular advancement appliances in the treatment of OSA has

Address for correspondence

Lt Col B Jayan

Classified specialist in Orthodontics

Army Dental Centre(R\&R), Delhi Cantt

New Delhi-110010

E mail jayan_deepa@rediffmail.com been documented by Cochrane collaboration as the highest level of scientific evidence[4].Most studies with mandibular advancement appliances exclude the patients with severe OSA and include patients who failed other treatment modalities with significant source of bias[5]. We have observed acceptable therapeutic efficacy with fixed mandibular advancement appliances in a few severe OSA patients[6,7].

Hence we carried out a pilot study with Thornton adjustable positioner (TAP), a titratable mandibular advancement appliance. TAP is an oral mandibular repositioning appliance. It alleviates snoring and sleep apnea by holding the mandible forward during sleep and prevent the soft tissue of the throat and tongue from collapsing into the airway and suffocating the patient during sleep. The basic principle of TAP is based on Cardio pulmonary resuscitation. The airway must be open and unobstructed to allow air to pass through the throat.

The aim of the present study was to evaluate therapeutic efficacy in severe OSA cases where apnea hypopnea index (AHI) is equal or more than 30/ sleep 
hour. The objectives were to compare AHI and ESS (Epworth sleepiness scale) score before and after prescribing TAP.

\section{Patients and methods}

12 adult patients fulfilling the following criteria were assessed for therapeutic efficacy with TAP in this prospective study .Patient data is represented vide Table 1.

Table 1: Patient Data

\begin{tabular}{|l|l|l|l|l|l|l|}
\hline Case No & Age & Sex & Pre AHI & Post AHI & Pre ESS & Post ESS \\
\hline 001 & 35 & M & 64.0 & 28.6 & 15 & 8 \\
\hline 002 & 54 & F & 46.7 & 17.4 & 14 & 9 \\
\hline 003 & 65 & M & 43.9 & 14.6 & 12 & 8 \\
\hline 004 & 50 & F & 42.3 & 20.1 & 14 & 9 \\
\hline 005 & 65 & M & 33.9 & 19.8 & 12 & 10 \\
\hline 006 & 46 & M & 54.0 & 12.2 & 14 & 06 \\
\hline 007 & 40 & M & 48.7 & 29.4 & 09 & 07 \\
\hline 008 & 52 & F & 43.6 & 17.9 & 11 & 9 \\
\hline 009 & 44 & M & 49.0 & 26.4 & 14 & 10 \\
\hline 010 & 50 & M & 50.2 & 19.7 & 12 & 9 \\
\hline
\end{tabular}

\section{Inclusion criteria:}

- Polysomnography (PSG) diagnosed OSA patients where AHI( Apnea-Hypopnea index) was equal to or more than 30/sleep hour.

- Body mass index $(\mathrm{BMI})</=30 \mathrm{Kg} / \mathrm{m} 2$

- Patients with at least one of the following features on the lateral cephalogram.

Reduced posterior airway space

Increased hyoid distance

Decreased SNB angle and increased ANB angle

\section{Exclusion criteria}

- Primary central apneas and hypopneas

- Anatomically caused upper airway obstruction

- Pre existing Temporo mandibular pain/ dysfunction

- Maximum mandibular protrusion less than $5 \mathrm{~mm}$

- Bilateral distal edentulousness, Complete edentulousness

- Advanced periodontal disease with multiple mobile teeth.

The case details were recorded on a sleep disordered breathing examination form which was inclusive of BMI, neck size, alcohol consumption and sedative usage details, sleep position, frequency and intensity of snoring, Epworth sleepiness scale (ESS), tongue size, airway grading, periodontal examination, TMJ evaluation, blood pressure history, maximum mandibular protrusion and clearance between central incisors at maximum opening.

All patients were subjected to lateral cephalograms which were recorded at end expiration and patients were instructed not to swallow during radiography. All cephalograms were manually traced and basic cephalometric evaluation was performed in all the cases.

\section{Fabrication of TAP and titration}

Maxillary and mandibular impressions were made with sodium alginate impression material and working models were made with dental stone. The required mandibular protrusion was recorded with George Bite gauge using medium body rubber base registration material. The registration was recorded at $50 \%$ of maximum mandibular protrusion [8 ] (Fig 1). The TAP is a two split system in which titration unit is placed anteriorly consisting of stainless steel screw with hook on the upper jaw splint and transverse bar on the lower jaw splint (Fig $2,3,4)$. The splint bases were vacuum formed (Biostar) and were made from thermoplastic hard soft composite material (Dura soft $2.5 \mathrm{~mm}$ ). The titration was done incrementally by activating the knob of the screw clockwise resulting in the forward movement of the lower jaw splint. The titration was conducted on weekly basis till the patient reported good improvement subjectively. However following this protocol of titration we did not exceed $70 \%$ of maximum mandibular protrusion.

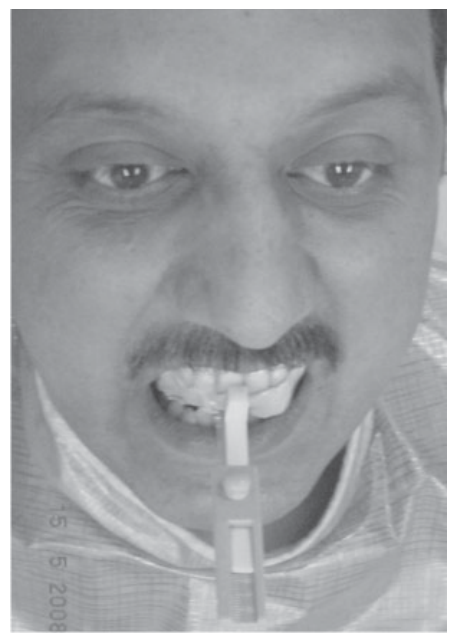

Figure 1: Bite recording for mandibular advancement appliance with George bite gauge 


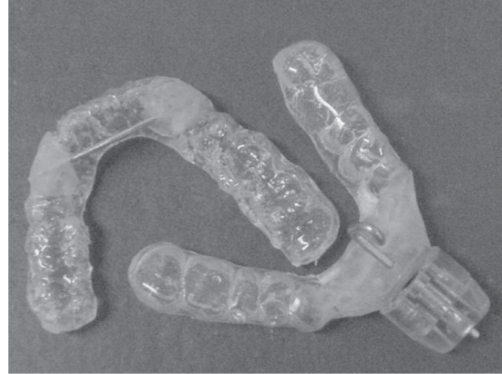

Figure 2: Thornton adjustable positioner

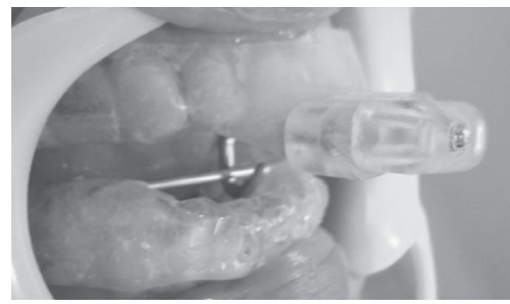

Figure 3: Lateral view of TAP when placed in the mouth

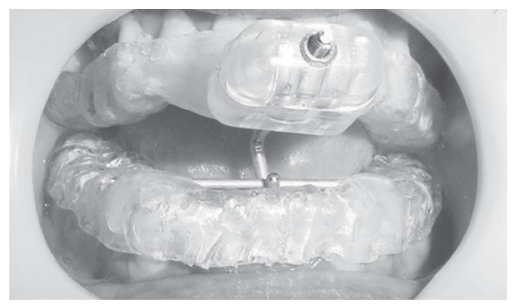

Figure 4: Frontal view of TAP when placed in the mouth

PSG was done with TAP in situ after completion of titration for objective assessment. The titrating screw was replaced with locking plates (Fig 5). Pre and post AHI and ESS (Epworth sleepiness scale) scores were compiled and suitable statistical tests applied.

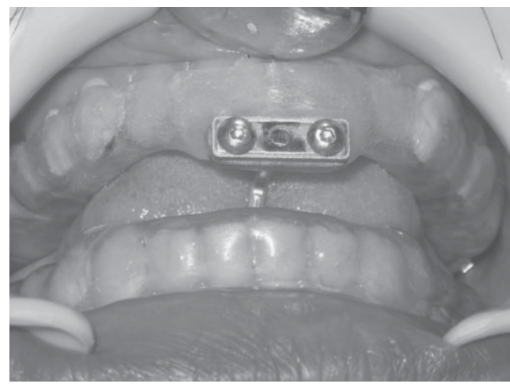

Figure 5: Titrating screw of the TAP has been replaced with locking plates following adjustments

\section{Results}

Of the twelve cases of severe OSA included in the present study two were loss to follow up ( $\mathrm{n}=10)$.Comparison of pre AHI scores( diagnostic PSG) with post treatment
AHI scores (PSG with OA in situ) decreased from 47.63 $\pm 7.93($ mean \pm SD) to $20.61 \pm$ 5.78.A highly significant improvement in AHI scores was observed (Table 2).56. $73 \%$ improvement in AHI scores was observed. Comparison of pre and post ESS scores showed a mean decrease from $12.70 \pm 1.82$ to $8.50 \pm 1.26$ (Table 2). Two patients complained of pain in the TMJ and masticatory muscles during the first week with TAP therapy. Three patients complained of excess salivation in the initial weeks. In 8 cases, mandible was titrated to $70 \%$ of maximum protrusion.

Table 2 : Comparison of Pre and Post AHI and ESS Scores

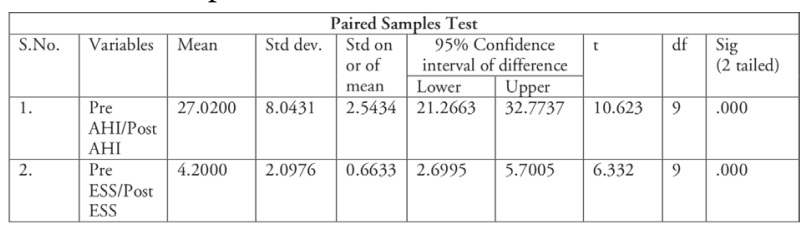

\section{Discussion}

Custom made mandibular advancement devices are an effective treatment option for snoring, upper airway resistance syndrome and obstructive sleep apnea. Evidence based data indicates their efficacy, and international sleep societies recommend oral appliance therapy for patients with sleep related breathing disorders $[1,2,4]$.

The use of oral appliances in treating OSA was first described in 1982 [9]. The therapeutic effect of mandibular advancement device was first documented in 1984 [10]. Adjustable mandible advancing oral appliances became the predominant form of oral appliance therapy for OSA in the 1990s [11,12 ]. Since then various titratable mandibular advancement appliances have been introduced which vary significantly in design however the philosophy remains the same. A mandibular advancement appliance should positively place the lower jaw in a predictable and maximally therapeutic position. During sleep, and whenever the person is in the supine position, the mandible tends to move inferiorly and posteriorly. The vertical mandibular opening during sleep is larger in patients with OSA than in healthy adults [13]. The vertical mandibular posture is associated with a decreased pharyngeal diameter and an increase in upper airway resistance [14]. Studies with fiber optic endoscopy, computerized tomography and MRI have demonstrated that patients with OSA have an elliptical upper airway shape with long axis oriented in the saggital plane $[15,16]$.

Indian Journal of Sleep Medicine (IJSM), Vol. 3, No. 3, 2008 
TAP appliance was developed by Dr N Keith Thornton which involves construction of separate appliance for maxilla and mandible. A separate appliance for each jaw makes fitting easier and makes it more difficult to dislodge because the removal of the appliance is in different path of opening. Connecting the upper and lower appliance is accomplished by a single hook and latch in the anterior region.

Because of the versatility and ease of adaptation the TAP is more effective than the one piece appliance .The TAP design restricts all backward movements while still allowing the patient to move the mandible forward and side to side as well as open the mouth if necessary. The patients often complained of inability to close the mouth while sleeping due to the presence of the titrating screw. But once the desired subjective and objective improvements were observed the titrating screw was removed and replaced with locking plates which enabled patients to close the lips.

A study reported by Pancer J and co-workers concluded TAP to be an effective treatment alternative for selected patients with snoring and OSA including severe OSA [17]. In this study the base line AHI was 44 $\pm 28 / \mathrm{h}$ which is comparable with the present study which recorded $47.63 \pm 7.93 / \mathrm{h}$. The improvement in AHI observed was $10 \pm 9 / \mathrm{h}$ when compared to $20.61 \pm 5.78 / \mathrm{h}$ in the present study. This is probably due to the sample size who underwent PSG with TAP $(n=61)$ and varying degrees of OSA was considered. A predictable AHI based results was achieved with TAP in a case control study which encompassed examination of initial effects of PSG in patients with OSA [18 ].However in this study the baseline AHI was $19.2 \pm 12.8 / \mathrm{h}$ and improved to $3.3 \pm 7.8 / \mathrm{h}$.

Based on scientific appraisals and international guidelines most of the sleep societies recommend oral appliance treatment for primary snoring ,upper airway resistance syndrome, mild to moderate OSA (AH1 up to $30 / \mathrm{h}$ ] and in severe cases, not amenable to CPAP therapy. The above thesis was supported in a study by Marklund and colleagues who specifically evaluated the efficacy of mandibular advancement devices in OSA patients with varying levels of severity of OSA [19 ].In this prospective study they demonstrated a significant reduction in AHI in patients with wide range of OSA severities but the greatest improvement was observed in patients with mild and moderate cases. In influential review by Kathleen Ferguson on oral appliances (OA) therapy for OSA has pointed out that most studies exclude the patients with severe OSA and include patients who failed other treatment modalities, thus there exists a significant source of bias [20].

A randomized clinical trial in 2002 concludes that mandibular advancement appliance was less effective in subjects with most severe OSA. ( pre treatment $\mathrm{AHI}>50$ ) [21]. In contrast a study by Henke and colleagues of 28 patients with OSA showed that severity of OSA and the site of airway closure did not predict the efficacy of the device. In this study patients with severe sleep apnea (mean AHI score of 53/hr) had a mean reduction of 53 $\%$ in their AHI scores [22]. We have also observed good improvement in severe OSA cases treated with fixed mandibular advancement appliances [6,7].

Therefore we are of the opinion that variables of polysomnographic severity of OSA and the site of airway closure should not be used to exclude patients from receiving treatment with oral appliances. Therapeutic goals should be to ameliorate the daytime symptoms of OSA and to lower the AHI scores by $50 \%$ to optimize oxygen saturation.

A publication of Uppsala University concluded that mandibular advancement appliance was effective in mild, moderate and severe cases [23]. They observed that severe OSA cases might benefit from more pronounced advancement $(75 \%)$ compared with a short degree of advancement (50\%). We have also observed acceptable therapeutic efficiency with oral appliances in severe OSA cases. The appliances in our reported case series were recorded at $70 \%$ of maximum mandibular protrusion $[6,7]$.

There are paucity of studies with regard to prescribing oral appliances with definite inclusion and exclusion criteria. We have observed predictable cephalometric measurements in OSA cases in a study on urban Indian obese and non obese OSA cases in 2007 [24]. So we factored predictable cephalometric findings and BMI prior to prescribing TAP. In the present prospective case series we have observed significant $(\mathrm{p}<001)$ improvement AHI and ESS scores. We also observed $56.73 \%$ improvement in AHI scores. Therefore we are of the opinion that if cephalometric findings like reduced posterior airway space/ retroglossal space, increased hyoid-mandibular plane distance, retrognathic mandible and $\mathrm{BMI}</=30 \mathrm{Kg} / \mathrm{m} 2$ are factored into, along with established dental norms for oral appliance therapy then, with $70 \%$ mandibular protrusion with TAP desirable 
therapeutic efficacy can be achieved in severe OSA cases. We recommend multi centric trials to validate the proposed criteria for prescribing titratable mandibular advancement appliances in cases of severe OSA.

\section{Conclusion}

The present study has observed significant improvement subjectively and objectively in severe OSA cases treated with titratable mandibular advancement (TAP). This may be due to the fact that we factored predictable cephalometric measurements, BMI $\leq 30 \mathrm{Kg} / \mathrm{m} 2$ and mandibular protrusion of $70 \%$ into the protocol for prescribing oral appliance.

\section{References}

1. American Academy of Sleep Medicine (1995) Practice parameters for the treatment of snoring and obstructive sleep apnea with oral appliances. Sleep 18:501-510. (Pub Med)

2. An American academy of sleep medicine report. Practice parameters for treatment of snoring and obstructive sleep apnea with oral appliances. An update for 2005. Sleep2006. 29.240-243.

3. Schwarting S, Huebers $U$, Heise M, Schlieper J, Hanschuld A. Position paper on the use of mandibular advancement devices in adults with sleep related breathing disorders. Sleep breath. 2007; $11(2): 125-126$.

4. Lim J, Lasserson TJ, Fleethan J, Wright J. Oral appliances for obstructive sleep apnea. Cochrane database syst Rev. 2006; CD004435.

5. Ferguson K. Oral appliances therapy for obstructive sleep apnea. Am J Resp. Crit Care Med. 2001;163(6):12941295.

6. Jayan B, Prasad BNBM, Rajput AK, Bhattacharya R, Dhiman RK, Management of a case of severe obstructive sleep apnea with acrylic herbst splint appliance. Indian Journal of Sleep Medicine 2006;1(2). 157-160.

7. Jayan B, Prasad BNBM, Rajput AK, Bhattacharya R, Dhiman RK, Thampi PS. Management of obstructive sleep apnea with oral appliances: our experience. Indian Journal of sleep medicine. $2006 ; 1(4): 204-207$.

8. Clark WJ. Bite registration in Twin block technique. In Twin block functional appliance therapy: Applications in Dentofacial orthopaedics. Mosby-Wolfe. 1995 ; 25-26.

9. Cartwright RD, Samelson CF. The effects of non surgical treatment for Obstructive sleep apnea. The tongue retaining device. JAMA. 1982;248:705-709.

10. Meyer-Ewert K, Schafer H, Klob W. Treatment of sleep apnea by mandibular protracting device. 7th European
Congress of sleep research. Munchen.217.

11. Lowe AA. Dental appliances for snoring and obstructive sleep apnea. In Kryger M, Roth T, Dement W (eds): Principles and practice of sleep medicine, ed 3. Philadelphia,WB Saunders, 2000; 929-939.

12. Schmidt NW. Recent developments in oral appliance therapy of sleep disordered breathing. Sleep and breathing. 1999;3.103-106.

13. Myamoto K, Ozbek MM, Lowe AA et al. Mandibular posture in sleep in patients with obstructive sleep apnea. Arch Oral Biol. 1999; 44: 657-664.

14. Kuna ST, Remmers JE. Neural and anatomical factors related to upper airway occlusion during sleep. Med Clinc North Am. 1985;69:1221-1242.

15. Schwab RJ, Gefter WB, Hoffman EA, Gupta KB, Pack Al. Dynamic airway imaging during awake respiration in normal subjects and patients with sleep disordered breathing.Am Rev Respir Dis. 1993;148:1385-1400.

16. Rodenstein DO, Dooms G, Thomas Y, et al. Pharyngeal shape and size in healthy subjects, snorers and patients with obstructive sleep apnea. Thorax 1990; 45 : 722-727.

17. Pancer J, Al-Faifi S, Al-Faifi M, Hoffstein V. Evaluation of variable mandibular advancement appliance for treatment of snoring and sleep apnea. Chest.1999; 116(6): 1511 1518.

18. Rose EC, Germann M, Sorichter S, Jonas IE. Case control study in the treatment of Obstructive sleep disordered breathing with mandibular protrusive appliance. J Orofac Orthop. 2004; 65: 489-500.

19. Marklund $\boldsymbol{M}$, Satilin $C$, Sterlund $H$, Persson $M$, Franklin KA. Mandibular advancement device in patients with obstructive sleep apnea : long term effects on apnea and sleep. Chest 2001;120(1):162-9.

20. Ferguson K. Oral appliance therapy for Obstructive sleep apnea. Am J Resp Crit Care Med. 2001; 163 (6): 12941295.

21. Johnston CD, Gleadhill IC, Cinnamond MJ, Gabbey J, BurdenDJ. Mandibular advancement appliances and Obstructive sleep apnea: a randomized clinical trial. Eur J Orthod. 2003;.24(3): 251-262.

22. Henke K, Frantz D, Kuna S. An Oral elastic mandibular advancement device for obstructive sleep apnea. An J Respir Crit Care Med 2000;161:420-425.

23. Engstrom W, Louise $M$. Treatment effects with amandibular advancement appliance and Uvulo-pharyngoplasty in Obstructive sleep apnea : randomized control trials.Doctorol thesis. Uppsala university publications. 2003. (publications.uu.se/abstract.xsql? dbid=3376)

24. Jayan B,Prasad BNBM, Kotwal A, Kharbanda OP, Roy Chowdhury SK, Gupta SH. The role of cephalometric analysis in obese and non obese urban Indian adults with obstructive sleep apnea syndrome: A Pilot study. Indian J Sleep Med. 2007; 2(2): 59-63.

Indian Journal of Sleep Medicine (IJSM), Vol. 3, No. 3, 2008 